\title{
Quantitive analysis of electric vehicle flexibility: A data-driven approach
}

\author{
N. Sadeghianpourhamami ${ }^{\mathrm{a}}$, N. Refa ${ }^{\mathrm{b}}$, M. Strobbe $^{\mathrm{a}}$, C. Develder ${ }^{\mathrm{a}}$ \\ ${ }^{a}$ Ghent University - imec, IDLab,Dept. of Information Technology, \\ Technologiepark Zwijnaarde 15, 9052 Ghent, Belgium \\ ${ }^{b}$ ElaadNL \\ Utrechtseweg 310, building B42, 6812 AR Arnhem, The Netherlands
}

\begin{abstract}
The electric vehicle (EV) flexibility, indicates to what extent the charging load can be coordinated (i.e., to flatten the load curve or to utilize renewable energy resources). However, such flexibility is neither well analyzed nor effectively quantified in literature. In this paper we fill this gap and offer an extensive analysis of the flexibility characteristics of $390 \mathrm{k}$ EV charging sessions and propose measures to quantize their flexibility exploitation. Our contributions include: (1) characterization of the EV charging behavior by clustering the arrival and departure time combinations that leads to the identification of type of EV charging behavior, (2) in-depth analysis of the characteristics of the charging sessions in each behavioral cluster and investigation of the influence of weekdays and seasonal changes on those characteristics including arrival, sojourn and idle times, and (3) proposing measures and an algorithm to quantitatively analyze how much flexibility (in terms of duration and amount) is used at various times of a day, for two representative scenarios. Understanding the characteristics of that flexibility (e.g., amount, time and duration of availability) and when it is used (in terms of both duration and amount) helps to develop more realistic price and incentive schemes in DR algorithms to efficiently exploit the offered flexibility or to estimate when to stimulate additional flexibility.
\end{abstract}

Keywords: Electric Vehicles, Flexibility Quantization, Smart Grid

Email addresses: nasrin.sadeghianpourhamami@ugent.be (N. Sadeghianpourhamami), nazir.refa@elaad.nl (N. Refa), matthias.strobbe@ugent.be (M. Strobbe),

chris.develder@ugent. be (C. Develder)

Preprint submitted to Elsevier

September 4, 2017 


\section{Introduction}

Partly because of environmental constraints, electric vehicles (EVs) are increasingly being adopted as an alternative for internal combustion engine (ICE) cars. However, the load from EVs may increase the peak to average ratio of demand and hence create a need for additional generation and network capacity.

15 That extra capacity would only be required to meet the increased peak demand and therefore is used very infrequently [1]. Integration of information technology into the power grid (in the smart grid paradigm) alleviates this challenge by enabling the exploitation of demand side flexibility to reshape the consumption to meet the supply or network constraints (i.e., by flattening demand or by balancing against renewable generation). Consequently, a substantial body of research has focused on proposing demand response (DR) algorithms to coordinate EV charging and establish their benefits (a review of various DR algorithms for charging coordination is given in [2, [3], 4], and [5]). However, one of the main limitations of such proposed DR algorithms is their potentially unrealistic assumptions about the EV owner behavior (e.g., time of availability of EV, sojourn times and the fraction of the sojourn time that is not spent for charging and is named idle time). To design an efficient and practical DR algorithm, it is necessary to accurately understand the flexibility stemming from EVs and how to influence it (through price based and incentive based schemes) to maximize so DR benefits. However, despite various efforts in proposing DR algorithms, EV flexibility characteristics as DR's main asset have not been quantitatively analyzed. We believe such analysis can pave the way to more realistic demand response schemes (price-based or incentive based DR) in order to facilitate EV integration in the grid and therefore is the focus of this paper.

35 1.1. Objectives and Contributions

Understanding the flexibility characteristics, the influencing factors, and the motivation for its exploitation is an inevitable part of designing a realistic DR algorithm. Flexibility, despite its apparent simplicity, is neither straightforward to analyze nor to quantify. 
We pursue two objectives in this paper. Our first objective is to perform an in depth analysis of the flexibility characteristics of EVs based on a reasonably large real-world dataset (which to the best of our knowledge amounts to the largest dataset reported in literature, see Section 2.1 for further details). Our second objective is to quantify the flexibility exploitation and identify how the observed flexibility is utilized for various objectives (e.g., load flattening and load balancing against renewable (energy) sources) and whether there is any typical pattern in its exploitation. More precisely, we aim to answer the following research questions:

1. Do EV owners have specific habits to charge their cars (e.g., taking their cars to a charging station at particular times of the day)? To answer this question, we characterize the EV charging behavior by clustering the arrival and departure time combinations, as such identifying three behavioral clusters in our EV charging data (Section 2.2).

2. Are the characteristics of the charging sessions (e.g., arrival, sojourn and idle times) sensitive to seasonal changes or weekdays? To address this question, we systematically analyze the characteristics of the charging sessions in each behavioral cluster on weekdays and weekends and across various seasons. We also characterize the flexibility stemming from the sojourn times of EVs that are longer than the time required to (fully) charge their battery (Section 2.3).

3. How is flexibility (in terms of amount, time and duration of the shifted energy) exploited? Which aspect of flexibility (time and duration of availability or amount of deferrable energy) is more useful at various times of the day? We address these questions by considering two case studies (i.e., load flattening and load balancing scenarios) to investigate to what extent the observed flexibility would be exploited. To do so, we propose two measures and an algorithm to quantitatively analyze when flexibility is used in terms of the EV load volume as well as amount of time the load is deferred(Section 3.3 and Section 3.4 ). 
Estimating the EV charging load to assess its impact on the power grid has been the primary focus of research in facilitating EVs integration to the grid. In initial studies, before the wide-spread use of EVs, probabilistic models of driving behavior (with conventional ICE cars) were used to characterize a 75 charging session. This was done by estimating arrival and departure patterns, energy requirements and the covered distance in between trips. For example Lampropoulos et al. 6] derive an EV charging data profile from statistical characteristics of the driving behavior of conventional ICE cars. Clement-Nyns et al. [7] base their analysis on extrapolation of non-EV car usage in Belgium. Paevere et al. 8 model the spatio-temporal impact of EV load based on a linked suite of models of future EV uptake, their travel and charging/discharging models. Grahn et al. [9] derive EV charging behavior from non-EV driving behavior in Sweden. Pashajavid et al. [10] derive the demand profile of EVs from traveling and refueling information of non-EV in Tehran, and a more recent study [11] estimates possible states of EVs, regarding their demand, location and connection period, based on synthetic data which mimics reality.

Later studies, when EV penetration had increased, relied on the availability of EV charging datasets to use data-driven approaches to model the charging behavior of EVs and assess their impact on the grid. For instance, Xydas et 90 al. [12] characterize the charging demand of EVs by statistically analyzing and clustering a dataset of $22 \mathrm{k}$ sessions in UK. Khoo et al. [13] derive the impact of EV charging on peak load based on around 5k sessions from an Australian field trial and establish the expected impact on the total power demand in 2032-33 for the state of Victoria. Brady et al. 114] use a probabilistic charging module to translate the travel patterns of EVs into the respective power demand of the vehicles. Quiròs-Tortòs et al. [15] and Navarro-Espinosa et al. [16] use the probability distribution of start charging time and energy demanded during a connection of charging sessions in a one-year EV trial in Ireland to obtain the EV load demand and assess their impact in the low voltage distribution grid. 100 The aforementioned works focus mainly on analyzing the impact of EVs on 
the load curve and do not provide any quantitative analysis of the flexibility characteristic of EV charging sessions. The objective of our analysis presented here rather is to quantify the flexibility of the EV load, and quantitatively study user behavior.

User modeling (not focusing on flexibility) has been the subject of earlier works to assess the influence of charging behavior of different user categories on the load curve. For example, Franke et al. [17] examine the psychological dynamics underlying charging behavior of EV users. Spoelstra [18] aims at understanding the charging behavior of EV users and the factors constituting such behavior. Khoo et al. 13] have modeled the charging sessions for households and EV fleets during weekends and on weekdays in terms of arrival times and energy demands. Quiròs-Tortòs et al. [19] produce probability distribution functions (PDF) of different charging features (e.g., start charging time) for both weekdays and week-ends based on 68k samples from 221 residential EV users. They further discuss the effects of the EV demand on future UK distribution networks. Similarly, Richardson et al. 20, produce PDF of connection times and daily energy requirements of EV based on the charging behavior of 78 users for a duration of 1 year. Helmus et al. [21] distinguish a priori defined different user types (residents, commuters, taxis, etc.) and characterize them in terms 120 of EV charging session start and end times and the associated energy needs. Similarly, Aunedi et al. 22] characterize the charging behavior and the demand diversity of two predefined user categories: residential users and commercial users. Instead of defining the user categories a priori, Xydas et al. [12] cluster the observed charging sessions into distinct types of behavior. They derive aggregate models for three specific geographical areas, characterized by different clusters of "typical EV charging demand profiles". Similar characterization of charging session timing is presented by Kara et al. 23]. Similar to [12] and [23] (but using different clustering technique), we cluster the EV charging sessions into behavioral clusters. However, our work differs from the aforementioned papers: instead of focusing on the impact of EVs on the load curve, we characterize the flexibility stemming from the EVs as well as how such flexibility 
is used (in terms of both amount and duration) to flatten the load or balance against renewable energy.

Quantification of demand side flexibility and assessing its impact on allegration. However, they characterize the flexibility only in terms of the amount 
of shifted energy and not the duration. We on the other hand provide a complete quantification of flexibility in terms of not only the deferrable amount but also time of availability and the deferrable duration. Furthermore, detailed analysis of how the flexibility is used is also missing in the literature. We thus present an extensive analysis on how flexibility is exploited (using our proposed measures) to meet two representative objectives: peak reduction and balancing against RES.

Note that this paper is a substantial extension of our work in 28] since we now offer a more extensive analysis of the charging session characteristics and investigate the effect of seasonal changes and weekends on the characteristics of the charging sessions. Additionally, in [28, we quantized flexibility as the maximal load that could be deferred for a specific duration at any time of the day, 175 independent of any DR scheme. In other words, our previous analysis showed the flexibility potential that is available for utilization and not the flexibility that would be utilized to meet various DR objectives. In this paper, we complement our previous flexibility potential analysis and propose measures to quantify the actually exploited flexibility under two DR schemes: load flattening and load balancing.

\section{Analysis of EV Charging Behavior}

In this section, we address the first two research questions raised in Section 1.1 Do EV owners have specific habits in terms of charging their cars? Are the characteristics of the charging sessions (e.g., arrival, sojourn and idle reasonably large real-world dataset which is explained next. 


\subsection{Dataset Description}

The data for our analysis was collected by ElaadNL1 between 2011 to 2015 from public charging infrastructure deployed throughout the Netherlands. The dataset has more than $1.5 \mathrm{M}$ charging sessions characterized by arrival time, departure time, charging duration, and total power consumption. The EVs in this dataset are privately owned cars and thus comprise a mixture of various and a priori unknown types, without further information on their driving behavior. For our analysis, we took the subset of sessions from 22nd Dec 2014 to 21st Dec 2015 (i.e., 387,524 sessions) to ensure the observed charging behavior is not dominated by (potentially distinctive) behavior of novice users, since by that date the system had been deployed a few years already. Moreover, in this period there were not substantial extensions of the charging infrastructure: the number of deployed charging stations remained almost constant through 2015. The selected horizon effectively covers the four seasons and hence facilitates analysis of seasonal influences.

\subsection{Clustering of Charging Session Times}

The first question we address is: are there any typical behaviors in terms of arrival and departure times in the dataset? To answer this question, we have plotted the data in 2D space in terms of arrival time vs. departure time as shown in Fig. 1] We then adopted DBSCAN [29] clustering to cluster the data in that 2D space.

DBSCAN clustering is a density based clustering algorithm and we deemed

\footnotetext{
${ }^{1}$ ElaadNL is the knowledge and innovation center in the field of charging infrastructure in The Netherlands, providing coordination for the connections of public charging stations to the electricity grid on behalf of 6 participating distribution system operators (DSOs). It also performs technical tests of charging infrastructure, researches and tests smart charging possibilities of EVs, and develops communication protocols for managing EV charging. The EV charging session data is available upon request for non-commercial research purposes, subject to signing an agreement. For more information, please contact Chris Develder (email: chris.develder@ugent.be)
} 


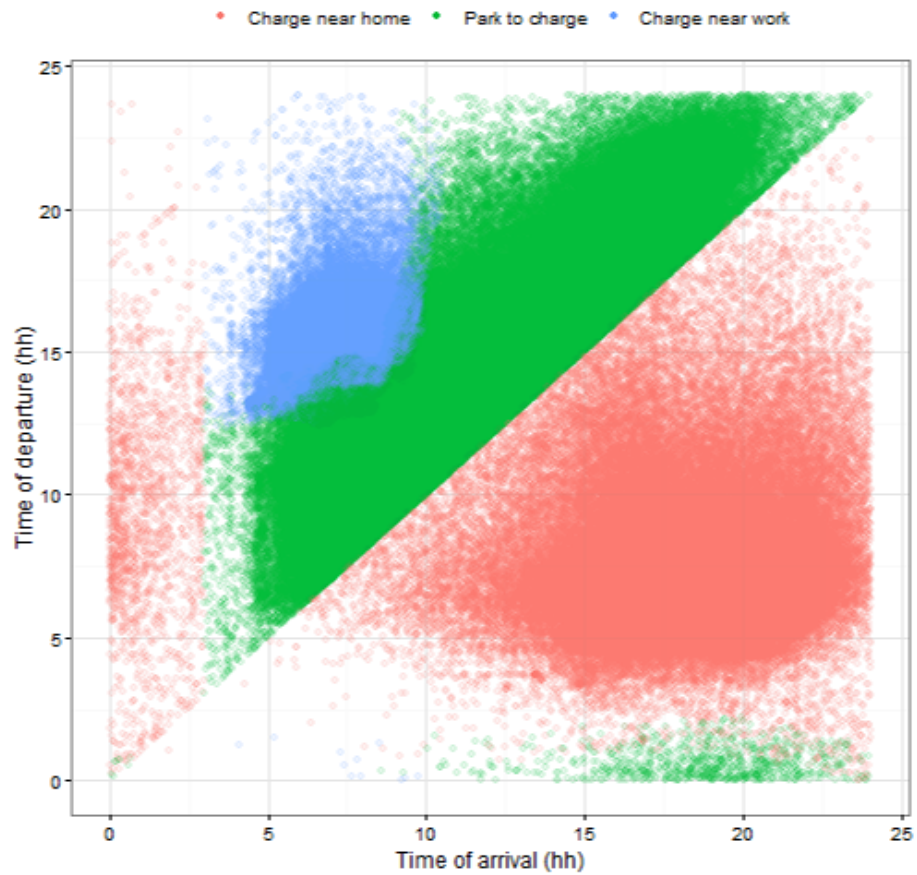

Fig. 1: behavioral clusters of sessions in terms of EV arrival and departure times. Both $X$ and $Y$-axis denote time-of-day (i.e., we report times as $t \bmod 24 \mathrm{~h}$ ): points below the $X=Y$ diagonal have departures on the day after the arrival or later. (Note that also some sessions plotted above the diagonal actually have departures $\geq 24 \mathrm{~h}$ after arrival)

it to be more suitable than other clustering algorithms (e.g., k-means and Gmeans [30]) for two reasons: (1) unlike k-means, DBSCAN does not require to a priori specify the number of clusters to distinguish and (2) DBSCAN is able to identify arbitrary shaped clusters without prior assumptions about the underlying distribution of data in each cluster, as opposed to the normal distribution assumed by the G-means clustering algorithm. One of the disadvantages of DBSCAN is its sensitivity to the parameters of the algorithm (i.e., $\varepsilon$, which specifies how close points should be to each other to be considered part of the same cluster; and minPts, which specifies the minimum number of points required to form a dense region). The values of $\varepsilon$ and minPts are empirically obtained from the data. To examine the sensitivity of DBSCAN to these pa- 
were able to identify 3 behavioral clusters in each month using similar values of $\varepsilon$ and minPts (i.e., $\varepsilon=0.4$ and $\operatorname{minPts}=90$ ).

Figure 1 shows the resulting behavioral clusters for the entire dataset. We named the clusters according to our interpretation of the observed behavior: charge near home, charge near work and park to charge clusters. The charge near home cluster (27.84\% of the total data) has arrivals in the afternoon/evening with departures mostly in the morning of the next/subsequent days. We hypothesize these are mostly people that live nearby the public charging station and park their car until they leave for work in the morning. Hence, the charging usually occurs at night for the sessions in this cluster. The charge near work cluster $(9.3 \%$ of the total data), which accounts for the smallest share of the data, is characterized by arrivals in the morning and departures in the evening. We assume these are people who either work near a public charging station or take their car to the station on their way to work (e.g., as a part of their this cluster has significantly smaller fraction of arrivals in weekends compared to the other two clusters (see Table 1 for fraction of weekend arrivals in each cluster). This type of behavior is absent in the datasets collected from residential charging (e.g., iMove [28]). The park to charge cluster (62.86\% of the total data) is the largest cluster and has arrivals/departures scattered throughout the day with sojourns that last not much longer than the time required to charge the battery. We hypothesize these are people that park specifically for the sake of charging the EV battery.

The aforementioned behavioral clusters provoke questions pertaining to what factors exactly distinguish them from each other, which we analyze next.

\subsection{Analysis of Behavioral Clusters: Weekdays and Seasonal Impacts}

In this section, we further analyze the sessions within each of the behavioral clusters in terms of their arrival time, sojourn time (i.e., how long the car is connected at the charging station) and idle time (i.e., the time between the 


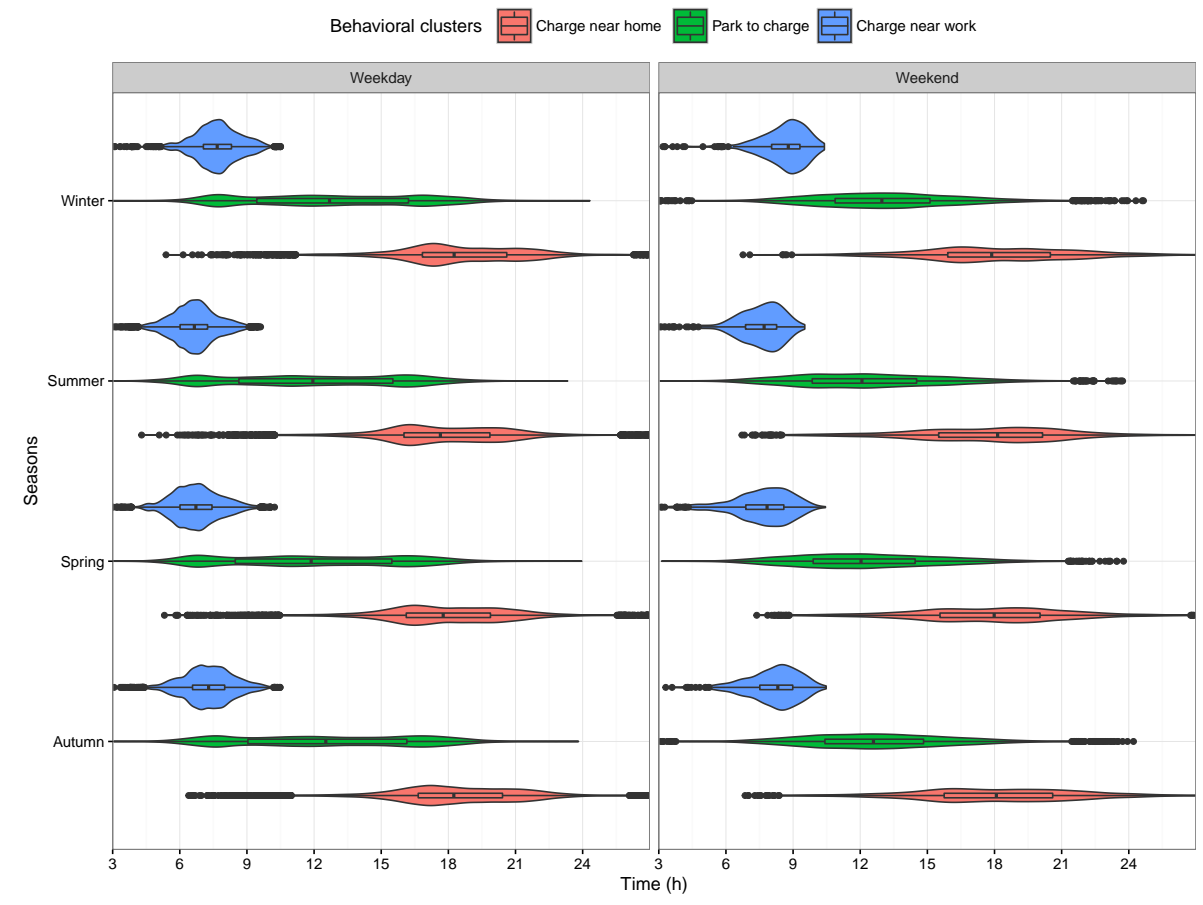

Fig. 2: Violin and box plots of time of arrivals for the behavioral clusters over weekends and weekdays in each season (Note that the reference is changed from midnight to $3 \mathrm{am}$ ( $2.30 \mathrm{am}$ to $3.30 \mathrm{am}$ is the interval with least number of arrivals) to acount for the fact that the activities right after the midnight are continuation of the late night activities)

completion of the charging and departure of the car). More formally, we define:

$$
\begin{aligned}
\text { Sojourn time } \triangleq \delta_{\text {sojourn }} & =t_{\text {depart }}-t_{\text {arrive }} \\
\text { Charging time } \triangleq \delta_{\text {charging }} & =t_{\text {end charging }}-t_{\text {start charging }}, \\
\text { Idle time } \triangleq \delta_{\text {idle }} & =\delta_{\text {sojourn }}-\delta_{\text {charging }}
\end{aligned}
$$

We also investigate the impact of weekends and seasonal changes on the aforementioned properties. 


\subsubsection{Analysis of Arrival Times}

Figure 2 shows the violin and box plots of arrival times for the behavioral

light and warmer weather in summer and spring. The interquartile ranges are 


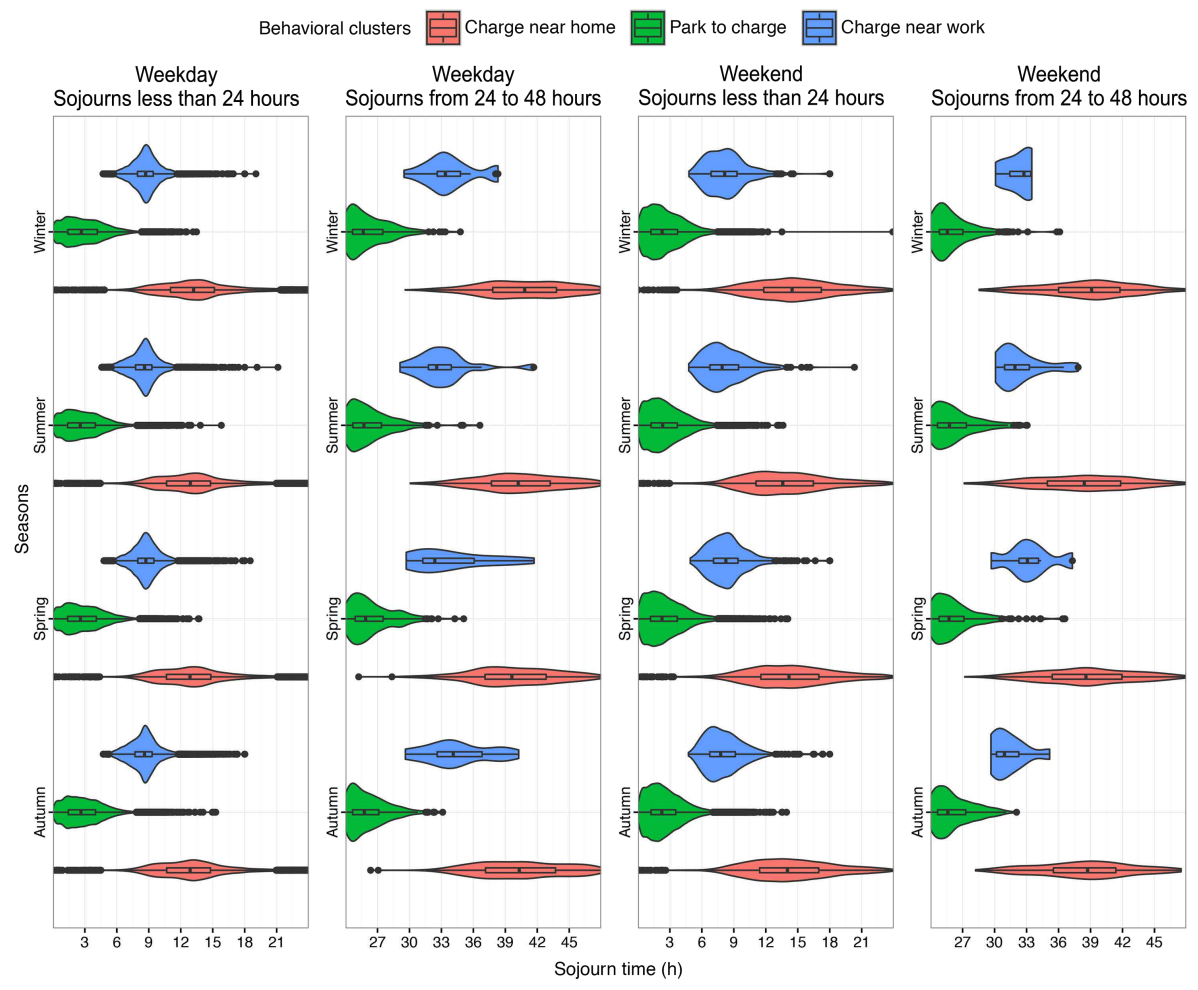

Fig. 3: Violin and box plots of sojourn times for the behavioral clusters over weekends and weekdays in each season

longer in weekends in all seasons. Seasonal changes do not significantly affect the interquartile ranges. Finally, arrivals are typically earlier during the weekdays of summer and spring but similar in all seasons during the weekends.

\subsubsection{Analysis of Sojourn Times}

Looking at each individual behavioral cluster, we observe that a minority of sessions have sojourn times of more than $24 \mathrm{~h}$ (see Table 1). We also find that for these clusters, the sojourn time distribution is multi-modal, where the modes correspond to subsequent days and are well separated. We thus partition the data into sub-clusters based on the departure time (i.e., depending on whether it is within the first, second, etc. period of $24 \mathrm{~h}$ following the arrival). Figure 3 shows the violin and box plots of sojourn times for the behavioral sub-clusters 
Table 1: Summary of cluster and sub-cluster fractions and average sojourn and idle times

\begin{tabular}{|c|c|c|c|c|c|}
\hline Cluster & $\begin{array}{l}\text { Weekend } \\
\text { arrival } \\
\text { fraction }\end{array}$ & $\begin{array}{l}\text { Sub-cluster } \\
\text { departures }\end{array}$ & $\begin{array}{l}\text { Sub- } \\
\text { cluster } \\
\text { fraction }\end{array}$ & $\begin{array}{c}\text { Mean } \\
\text { sojourn } \\
\text { time }\end{array}$ & $\begin{array}{c}\text { Mean idle } \\
\text { time }\end{array}$ \\
\hline \multirow{4}{*}{$\begin{array}{l}\text { Park to } \\
\text { charge } \\
(62.86 \%)\end{array}$} & \multirow{4}{*}{$28.24 \%$} & in $1^{\text {st }} 24 \mathrm{~h}$ & $98.9 \%$ & $2 \mathrm{~h} 28 \min$ & $48 \mathrm{~min}$ \\
\hline & & in $2^{\text {nd }} 24 \mathrm{~h}$ & $0.85 \%$ & $26 \mathrm{~h} 18 \mathrm{~min}$ & $22 \mathrm{~h} 48 \mathrm{~min}$ \\
\hline & & in $3^{\text {rd }} 24 \mathrm{~h}$ & $0.21 \%$ & $66 \mathrm{~h} 18 \mathrm{~min}$ & $62 \mathrm{~h} 42 \mathrm{~min}$ \\
\hline & & in $4^{\text {th }} 24 \mathrm{~h}$ and later & $0.11 \%$ & 105 h 24 min & $101 \mathrm{~h} 42 \mathrm{~min}$ \\
\hline \multirow{4}{*}{$\begin{array}{c}\text { Charge } \\
\text { near home } \\
(27.84 \%)\end{array}$} & \multirow{4}{*}{$23.24 \%$} & in $1^{\text {st }} 24 \mathrm{~h}$ & $95.09 \%$ & $13 \mathrm{~h} 24 \mathrm{~min}$ & $10 \mathrm{~h}$ \\
\hline & & in $2^{\text {nd }} 24 \mathrm{~h}$ & $3.44 \%$ & $39 \mathrm{~h} 36 \mathrm{~min}$ & $36 \mathrm{~h} 12 \mathrm{~min}$ \\
\hline & & in $3^{\text {rd }} 24 \mathrm{~h}$ & $0.9 \%$ & $63 \mathrm{~h} 48 \mathrm{~min}$ & $60 \mathrm{~h} 6 \mathrm{~min}$ \\
\hline & & in $4^{\text {th }} 24 \mathrm{~h}$ and later & $0.57 \%$ & 113 h 30 min & $109 \mathrm{~h} 54 \mathrm{~min}$ \\
\hline \multirow{4}{*}{$\begin{array}{c}\text { Charge } \\
\text { near work } \\
(9.3 \%)\end{array}$} & \multirow{4}{*}{$6.33 \%$} & in $1^{\text {st }} 24 \mathrm{~h}$ & $99.51 \%$ & $8 \mathrm{~h} 42 \mathrm{~min}$ & $5 \mathrm{~h} 30 \mathrm{~min}$ \\
\hline & & in $2^{\text {nd }} 24 \mathrm{~h}$ & $0.33 \%$ & $33 \mathrm{~h} 24 \mathrm{~min}$ & $29 \mathrm{~h} 12 \mathrm{~min}$ \\
\hline & & in $3^{\text {rd }} 24 \mathrm{~h}$ & $0.4 \%$ & $38 \mathrm{~h} 12 \mathrm{~min}$ & $34 \mathrm{~h} 24$ min \\
\hline & & in $4^{\text {th }} 24 \mathrm{~h}$ and later & $0.09 \%$ & 119 h 42 min & $115 \mathrm{~h} 18 \mathrm{~min}$ \\
\hline
\end{tabular}


over weekends and weekdays in each season. We only show the first 2 subarrivals) since the later sub-clusters constitute less than $1 \%$ of the data (see Table 1). In general, seasonal changes have minor effects on sojourn times in the behavioral clusters, but weekends impact the sojourn times more significantly. Further details about the weekend and seasonal impacts on the sojourn times are based on the 1 st sub-clusters (i.e., departures within 1st $24 \mathrm{~h}$ ) since in the second sub-clusters (i.e., departures within second $24 \mathrm{~h}$ ), distributions of the sojourn times have similar characteristics as ones in the first sub-clusters. One interesting characteristic is the approximate shift of $24 \mathrm{~h}$ in the average sojourn times in the second sub-clusters from the average values of the first sub-clusters (as seen from Table 1)

For the sessions in the charge near work cluster, the distribution of sojourn times are right-skewed in weekends and symmetrical or left-skewed during weekdays. This implies that typically the sessions have shorter sojourn times in weekends (average sojourn times are $8 \mathrm{~h} 18 \mathrm{~min}$ and $8 \mathrm{~h} 48 \mathrm{~min}$ for arrivals in weekend and weekdays respectively). Additionally, the interquartile ranges are smaller in the weekdays, implying a more predictable sojourn time. The largest interquartile range is in summer weekends.

Sessions in the park to charge cluster typically have smaller sojourn times than sessions in other clusters. As shown in Fig. 3. the distributions are left skewed for both weekend and weekdays, with slightly larger interquartile ranges during weekdays. This implies that sojourn times are typically shorter in weekends (average sojourn times are $2 \mathrm{~h} 36 \mathrm{~min}$ and $2 \mathrm{~h} 48 \mathrm{~min}$ for arrivals in weekend and weekdays respectively). The seasonal changes do not impact the distribu320 tions significantly in this cluster.

Sessions in the charge near home clusters have considerably larger sojourn times than the sessions in other clusters. The distribution of the sojourn times are symmetrical for both weekends and weekdays, with larger interquartile ranges in weekends. Unlike the other clusters, the charge near home sessions 


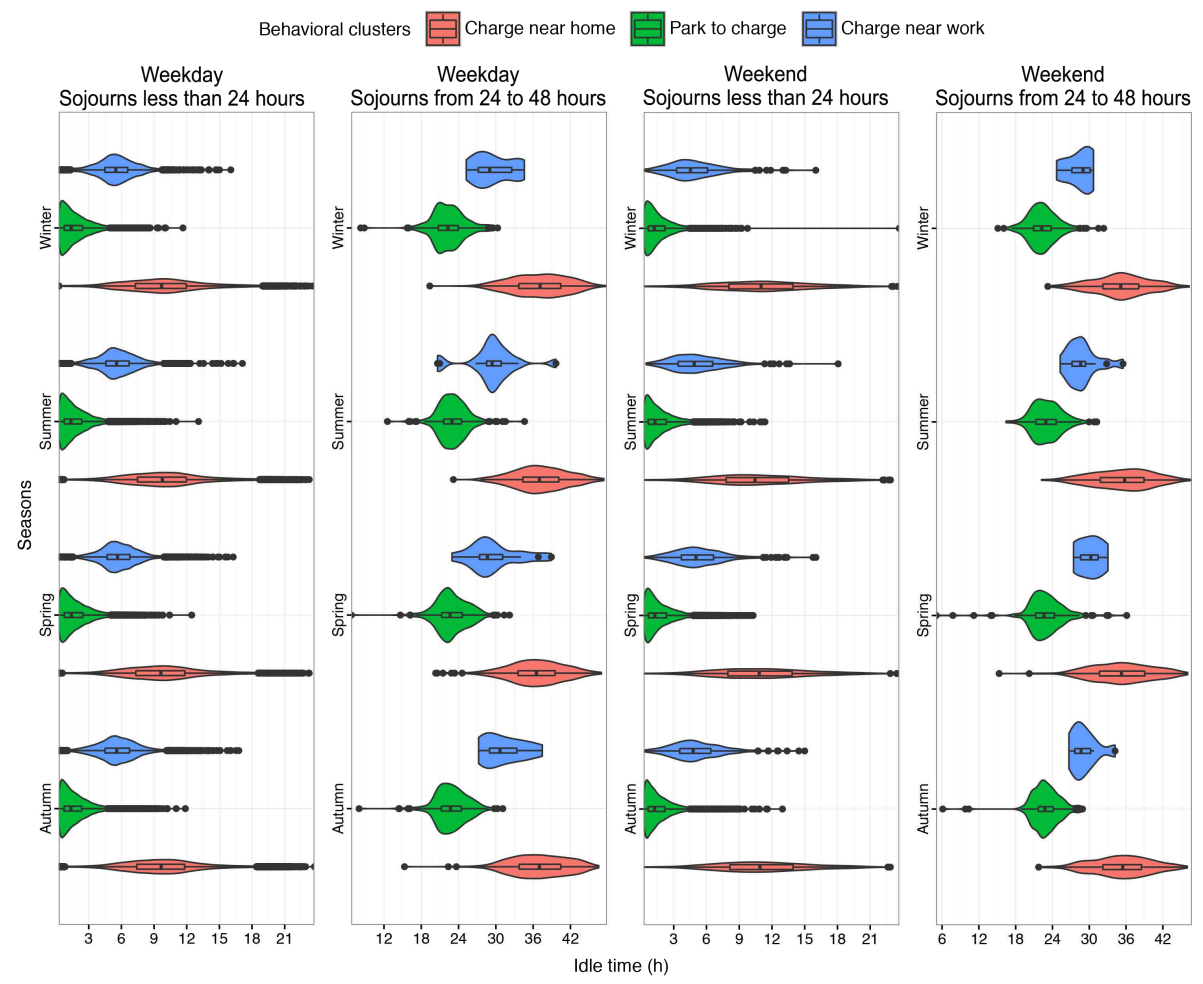

Fig. 4: Violin and box plots of Idle times for the behavioral clusters over weekends and weekdays in each season

have longer sojourns during weekends (the average sojourn times are $13 \mathrm{~h} 6 \mathrm{~min}$ and $14 \mathrm{~h} 18 \mathrm{~min}$ for arrivals in weekends and weekdays respectively). This is mainly because they are night time charging sessions, and people leave home later in the morning in the weekend.

\subsubsection{Analysis of Idle Times}

We have used the same sub-clustering approach to present the distribution of the Idle times in each behavioral cluster. Additionally, to improve the readability of the plots in Fig. 4, we have removed sessions with short idle times (i.e., less than $15 \mathrm{~min}$ ). This amounts to $43.08 \%$ and $33.58 \%$ of the data in 
weekends and weekdays respectively ${ }^{2}$ Note that the majority of the removed short idle times belong to the park to charge cluster. An overall view of Fig. 4 suggests that seasonal changes do not influence the distribution of idle times significantly, unlike weekend impacts, which are more apparent. Further details about the impact of the weekends on the distribution of the idle times are listed below.

The sessions in the charge near work cluster typically have 4 to $7 \mathrm{~h}$ of idle time in the first $24 \mathrm{~h}$ sub-cluster and 27 to $28 \mathrm{~h}$ of idle time in the second $24 \mathrm{~h}$ sub-cluster during the weekends. On weekdays, idle times are typically around 30 min longer than in weekends. On average (taking into account the sessions with short idle times), this cluster has $5 \mathrm{~h} 30 \mathrm{~min}$ of idle time in the first $24 \mathrm{~h}$ sub-cluster and $29 \mathrm{~h} 48 \mathrm{~min}$ of idle time in the second $24 \mathrm{~h}$ sub-cluster.

The sessions in the park to charge cluster typically have the shortest idle times, which suggests that the cars are usually parked with the motive of leaving as soon as the charging completes. The distribution of idle times are right skewed even after the removal of short idle times for the first sub-cluster over both weekends and weekdays. In the second sub-cluster, it looks symmetrical. On average, the park to charge sessions have $42 \mathrm{~min}$ of idle time in the first sub-cluster and $22 \mathrm{~h} 48 \mathrm{~min}$ of idle time in the second sub-cluster.

The charge near home sessions offer longer idle times (i.e., $10 \mathrm{~h}$ in the first and $36 \mathrm{~h}$ in the second sub-cluster) than the other clusters. The distributions of the idle times are symmetrical in all the sub-clusters and during both weekends and weekdays. The interquartile ranges span from $8 \mathrm{~h}$ to $14 \mathrm{~h}$ in the weekends and from $7 \mathrm{~h} 30 \mathrm{~min}$ to $12 \mathrm{~h}$ during the weekdays in first sub-cluster.

\section{Flexibility Quantization}

Our quantitative analysis of flexibility exploitation relies on the aforementioned EV charging data collected by ElaadNL, and renewable generation data

\footnotetext{
${ }^{2}$ However, the average values in Table 1 do include the short idle times in their calculation.
} 
Table 2: Nomenclature

\begin{tabular}{cl}
\hline \multicolumn{2}{l}{ Input parameters } \\
\hline$N$ & Total number of cars in the optimization window \\
$H$ & The length of the optimization window (the number of 15 min time slots) \\
$\gamma_{n h}$ & Maximum allowable energy consumption for car $n$ in slot $h$ \\
$E_{n}$ & Total energy to be scheduled for car $n$ \\
$P_{n}^{a v g}$ & Average power consumption of car $n$ \\
$\alpha_{n}$ & Arrival slot of car $n$ \\
$\beta_{n}$ & Departure slot of car $n$ \\
\hline Decision variables \\
\hline$x_{n h}$ & Energy scheduled to charge car $n$ in slot $h$ \\
$L_{h}$ & Total energy consumed in slot $h$ \\
\hline
\end{tabular}

obtained from ELIA (Belgium's electricity transmission system operator) $3^{3}$ The data obtained from ELIA comprises wind and solar energy generation measurements in 15 min intervals for the region of Flanders in Belgium. We rescaled the renewable energy production data to keep similar monthly wind to solar ratios as of the ones in Netherlands 4 Additionally, we further scaled the data to ensure the total yearly generation is similar to the total yearly demand of all the EV sessions considered in our study. We provide an assessment of flexibility exploitation in coordinated charging for two scenarios: (i) load flattening and (ii) load balancing against renewable production. As a reference, we take uncoordinated charging and refer to it as a business as usual scenario without flexibility exploitation.

Each time slot is characterized by a 15 min interval $h \in \mathbf{H}=\{1,2, \ldots, H\}$ and the EVs are denoted as $n \in \mathbf{N}=\{1,2, \ldots, N\}$. Table 2 summarizes all the

\footnotetext{
3 http://www.elia.be/en/about-elia

${ }^{4}$ See http://en-tran-ce.org/ for yearly reports of renewable generations in Netherlands.
} 
model parameters and the decision variables.

\subsection{Uncoordinated Charging: Bussiness as Usual}

In the business as usual (BAU) scenario, charging starts immediately upon arrival. In the ElaadNL dataset, vehicles are charged according to this BAU scenario and the charging time as well as the total energy consumption is reported for each session. The load in each time slot (i.e., of 15 min duration) is hence calculated as $P_{\text {slot }}=\Delta t \cdot E_{n} /\left(t_{B A U}-t_{\text {arrive }}\right)$, where $t_{B A U}$ is the time of the completion of charging in the BAU regime and $\Delta t$ is the duration (in hours) of each slot (i.e., $\Delta t=0.25 \mathrm{~h}$ ) in our settings.

\subsection{Coordinated Charging: Load Flattening and Load Balancing}

In the coordinated charging scenario, charging decisions are optimized by an aggregator to meet a predefined objective function. We formulate such a problem as a quadratic optimization (i.e., a quadratic objective function subject to linear constraints). To make the problem scalable and solvable in close to realtime, we define an optimization window of length $H=96$ time slots (i.e., 24 h) which starts at the present time slot (denoted as "Now") and moves one slot in

each iteration. We thus consider a receding horizon control approach, where we repeatedly solve the optimization problem to find the decision variables covering the window ("Now", "Now $+H$ ").

For load flattening, the objective function is defined as:

$$
\underset{\mathbf{L}, \mathbf{X}}{\operatorname{minimize}} \quad M \sum_{h=1}^{H} L_{h}^{2}+\sum_{n=1}^{N} \sum_{h=1}^{H} \beta_{n} x_{n h}
$$

The first term in (4) is a convex quadratic cost function and reflects the total load that needs to be minimized in the optimization window. We define a second term in (4) as a secondary objective which penalizes charging at later slots. This ensures that charging at earlier slots is preferred when permutations of charging decisions across different slots have the same cost. Note that we 
multiply the first term in (4) by $M$, a large constant, to have the first term dominate the second term in the objective function.

For load balancing, the objective function is defined as:

$$
\underset{\mathbf{L}, \mathbf{X}}{\operatorname{minimize}} \quad M \sum_{h=1}^{H}\left(L_{h}-L_{R G}\right)^{2}+\sum_{n=1}^{N} \sum_{h=1}^{H} \beta_{n} x_{n h}
$$

The first term in (5) models the imbalance using a convex quadratic function. Note that (similar to 31]) we account for negative imbalance to be as bad as positive imbalance. Similar to (4), the secondary objective function in (5) ensures earlier charging when charging at various slots has the same cost.

Both of the objective functions are subject to the following linear constraints:

$$
\begin{array}{ll}
L_{h}=\sum_{n=1}^{N} x_{n h} & \forall h \in \mathbf{H} \\
E_{n}-E_{a} \leq \sum_{h=1}^{H} x_{n h} \leq E_{n} & \forall n \in \mathbf{N} \\
0 \leq x_{n h} \leq \gamma_{n h} & \forall n \in \mathbf{N}, h \in \mathbf{H}_{n} \\
x_{n h}=0 & \forall n \in \mathbf{N}, h \in \mathbf{H} \backslash \mathbf{H}_{n}
\end{array}
$$

where,

$$
\mathbf{H}_{n}= \begin{cases}\left\{\alpha_{n}, \ldots, \beta_{n}\right\} & \beta_{n} \leq H \\ \left\{\alpha_{n}, \ldots, H\right\} & \beta_{n}>H\end{cases}
$$

and

$$
E_{a}= \begin{cases}P_{n}^{a v g} \cdot\left(\beta_{n}-H\right) & \beta_{n}>H \\ 0 & \text { otherwise }\end{cases}
$$

Constraint (6) ensures that the total load consumed in slot $h$ is equal to the summation of the loads from all the cars scheduled to charge in slot $h$ of the optimization window. Constraint (7) ensures that the charging demand (i.e., $E_{n}$ ) is fulfilled within the car's sojourn time. When a car departs within the optimization window, (7) becomes an equality constraint (i.e., equals $E_{n}$ ). Constraint (8) 
limits the energy consumption in each slot to the car's allowable consumption level and constraint (9) prohibits any charging outside the sojourn time.

\subsection{Measures for Quantification of Flexibility Utilization}

As outlined in Subsection 1.2, the demand response potential of EVs has already been studied to some extent, but how exactly the offered flexibility is exploited in real-world scenarios has not been well clarified in literature. In this section, we address this gap and offer a quantitative analysis of the flexibility exploitation of EVs using various measures. We first define the flexibility using 3 factors [32]: (1) the amount of deferrable energy (i.e., the amount of energy that can be delayed without jeopardizing customer convenience or quality of the task to be fulfilled), (2) the time of availability (i.e., the time at which a customer offers the flexibility for exploitation), and (3) the deadline/permissible duration to exploit the offered flexibility (i.e., the maximum allowable delay for the energy consumption).

We define the following measures to adequately quantize the EV flexibility exploitation:

1. Eflex (flexibility utilization in terms of Energy): fraction of the maximum energy that could be consumed beyond $t_{B A U}$. More formally,

$$
E f l e x=\frac{\text { Energy consumed beyond } t_{B A U}}{\text { Maximum possible energy consumption beyond } t_{B A U}}
$$

2. Tflex (flexibility utilization in terms of duration): fraction of the maximum delay beyond $t_{B A U}$. More formally,

$$
\text { Tflex }=\frac{t_{\text {coordinated }}-t_{B A U}}{t_{\text {depart }}-t_{B A U}}
$$

where $t_{\text {coordinated }}$ refers to the time of completion of charging in the coordinated charging regime.

The combination of Eflex and Tflex values quantizes the fraction of flexibility (in terms of time and amount) that was utilized for each charging session. For example, when Tflex $=$ Eflex $=1$, the energy consumption is deferred as much as possible (i.e., $t_{\text {coordinated }}=t_{\text {depart }}$ ) and the consumption beyond $t_{B A U}$ is at 


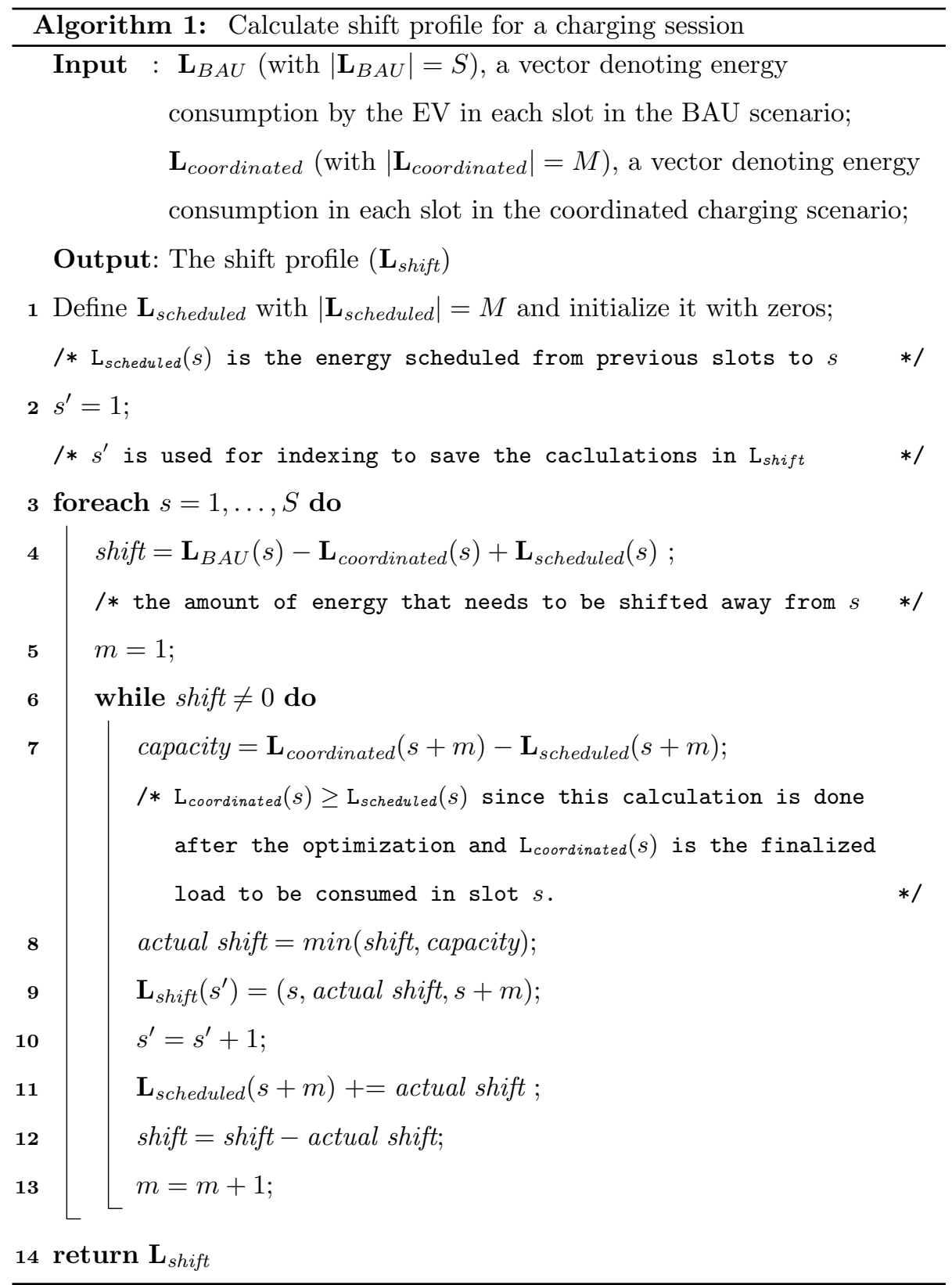

its maximal level. Another interpretation is that $1-$ Eflex is the fraction of 435 state-of-charge (SoC) at $t_{B A U}$ that has been realized in the flex scenario; for example, if $E$ flex $=0.25$, it means that at $t_{B A U}$, we have $1-0.25=75 \%$ of the 
desired SoC.

Although the aforementioned measures indicate how much of the offered flexibility is effectively utilized in each charging session, they do not provide information about the volume and the precise time shift of the deferred energy. Indeed, we believe it is interesting to know what portion of energy use is shifted to what time exactly. To quantitatively evaluate this, we define the shift profile of a charging session: the shift profile indicates how the energy is shifted from the BAU scenario to obtain the load pattern in the coordinated charging regime. In other words, it shows how much energy is shifted away from a particular slot and which slot it is scheduled to. We now explain how we calculate this shift profile, as outlined in Algorithm 1.

Given the $\mathbf{L}_{B A U}$ and $\mathbf{L}_{\text {coordinated }}$ vectors, respectively denoting the BAU and the coordinated energy consumption values in each slot, Algorithm 1 returns a $\mathbf{L}_{\text {shift }}$ list as its output. Each element of $\mathbf{L}_{\text {shift }}$ is a triple, depicting how much energy was shifted away from a particular slot and which slot it was shifted to (e.g., if $5 \mathrm{kWh}$ of energy is shifted from slot 1 to slot 3 , then the triple will have the following form: $\left.\left(s_{\text {from }}, E_{\text {shifted }}, s_{t o}\right)=(1,5,3)\right) !^{5}$ The algorithm starts by initializing $\mathbf{L}_{\text {scheduled }}$, a vector that keeps track of the amount of energy scheduled in a particular slot from the other slots (Line 1). For each slot $s$, starting with the first one, the amount of energy we need to shift away from it (i.e., shift) is calculated in Line 4 Note that to calculate the shift in each slot, we take the difference in energy consumption in the BAU and the coordinated charging scenario. Additionally, since any energy scheduled to be consumed in a slot also contributes to the delay of the energy consumption from that slot, we add the $\mathbf{L}_{\text {scheduled }}$ to the subtraction term. In the while loop, the shift is allocated to the subsequent slots following $s$, based on their available capacity. The amount of the allocated energy and the slot number is saved in $\mathbf{L}_{\text {shift }}$ (Line 9 and $\mathbf{L}_{\text {scheduled }}$ is updated accordingly (Line 11).

\footnotetext{
${ }^{5}$ Note that there could be several feasible shift profiles (e.g., $(1,1,3)$ vs. $\{(1,1,2),(2,1$, 3)\}) but here we calculate the one with minimal $s_{t o}-s_{\text {from }}$.
} 


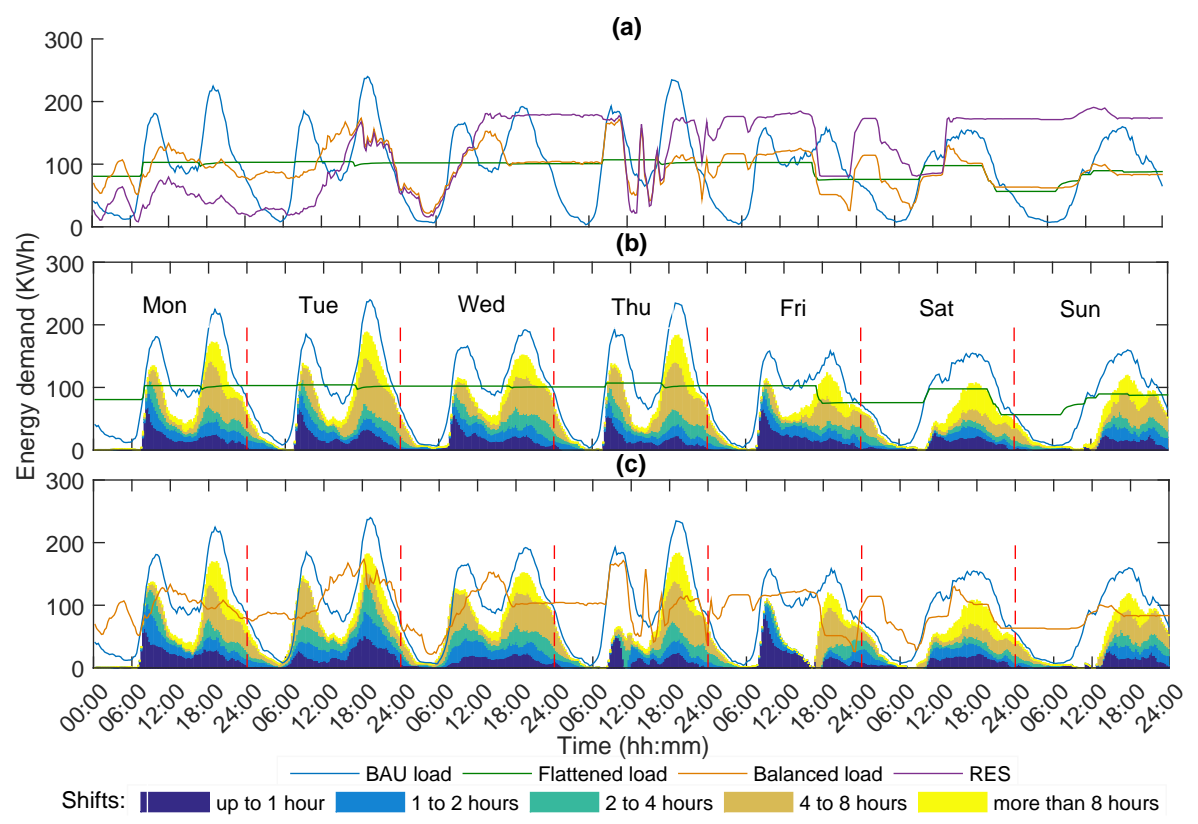

Fig. 5: (a) Load and renewable generation patterns from 5th to 11th Jan, (b) Amount of energy that is shifted away from each slot(for arrivals from 5th to 11th Jan 2015) in load flattening scenario and (c) Amount of energy that is shifted away from each slot (for arrivals from 5th to 11th Jan 2015) in load balancing scenario.

\subsection{Evaluation of Flexibility Exploitation}

In this section, we evaluate the flexibility exploitation using the measures and the algorithm proposed in the previous subsection. We implemented the optimization problem using MOSEK $\sqrt{6}$, in a MATLAB runtime environment.

Figure 5 shows how much energy (kWh) has been pushed away, and for how long, from BAU consumption, assuming 15 min long time slots in the optimization of the coordinated charging scenarios (i.e., load flattening and load balancing). A week long duration is selected for demonstration in Fig. 5 . Figure 5a shows the energy consumption patterns (in the BAU, load flattening and load balancing scenarios) and the scaled renewable generation in each slot of the

${ }^{6}$ MOSEK is a software package for solving mathematical optimization problems, see https: //www.mosek.com/ 
475 consumption patterns are multi-modal with distinct morning (around 9 am) and evening (around $8 \mathrm{pm}$ ) peaks on weekdays. During the weekends, the peak-toaverage ratio is lower than on weekdays and energy consumption patterns have a small peak around noon and a larger peak around $6 \mathrm{pm}$.

480

In the load flattening scenario (i.e., Fig. 5b), we observe the following:

1. The flexibility utilization is influenced by the BAU energy consumption patterns as well as the car arrival times (note that the arrival times and the BAU energy consumption patterns are also highly correlated.)

2. During weekdays: The load is typically shifted away from the morning peak (around 8-10 am) towards the afternoon valley (around 12-2 pm). Since the afternoon valley is not long away from the morning peak, the duration of the shift is typically lower compared to the shift from the evening peak to the midnight valley. Hence, we see more shifts of "up to 1 hour" long and less shifting of beyond " 4 hours" from the morning peak. On the other hand, the shifts from the evening peaks are longer to fill up the night valley, which is deeper and further away.

3. During weekends: The shifts from the evening peak to the night valleys are longer in weekends (typically more than 8 hours from the Saturday evening peak and more than 4 hours from the Sunday evening peak). The longer shifts from Saturday peaks are due to the wider and deeper valley between Saturday and Sunday peaks.

In the load balancing scenario, clearly the flexibility utilization is not only influenced by BAU energy consumption pattern and the car arrival times, but also by the renewable generation patterns. The flexibility exploitation for load balancing is depicted in Fig. 5f with the following key observations:

1. Although the flexibility utilization is not as consistent as for the load flattening scenario, still, longer shifts are observed in the evening peaks on weekdays. Additionally, there are still longer shifts from the Saturday peaks compared to the shifts from the Sunday peaks.

2. In general, longer shifts from the evening peaks are observed when there 

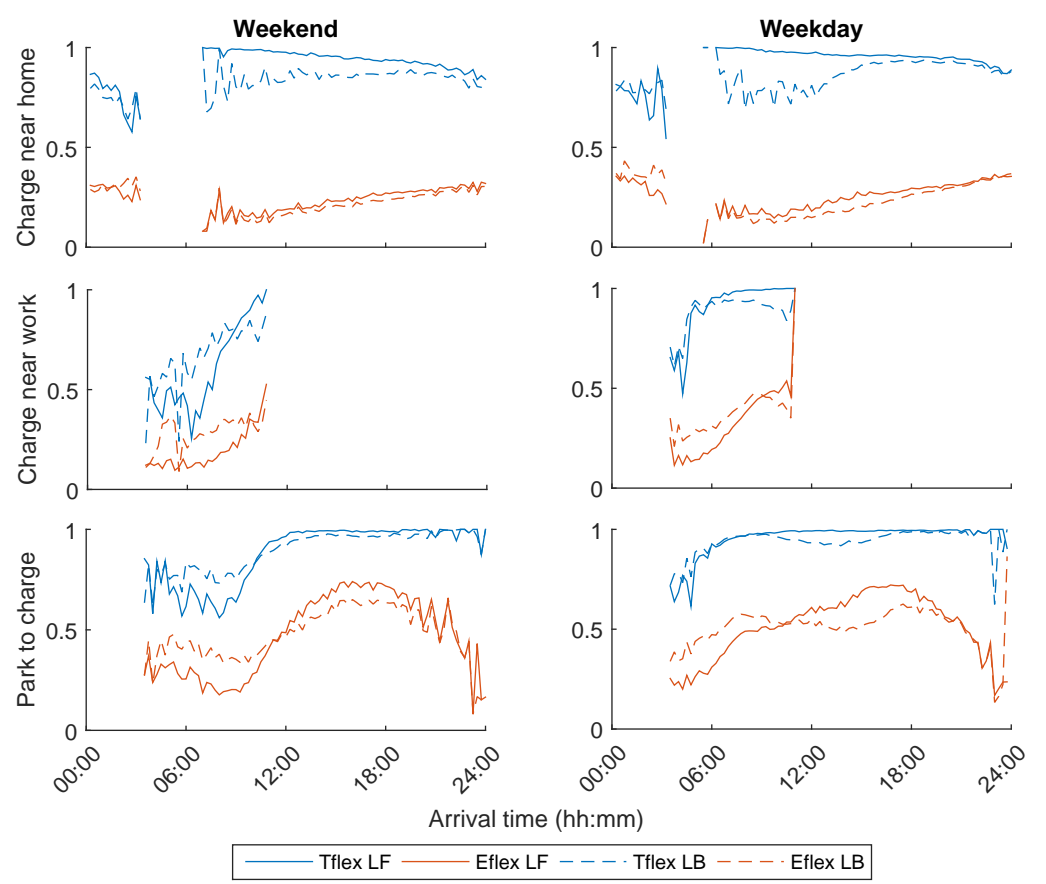

Fig. 6: Average Tflex and Eflex values for each 15 min long timeslot in a day (LB: load balancing, LF:load flattening)

is substantial renewable generation in the night valleys.

The observations based on Fig. 5give insight in the motivation for utilization of the flexibility and, hence, how much energy is required to be shifted and for how long. This is particularly useful for price-based or incentive-based demand response programs aiming to influence the offered flexibility at various hours of the day accordingly (using a relevant price or incentives). For example, the longer shifts from morning peak are not as frequent as the ones from the evening peak and hence, a lower incentive could be given for longer sojourn time of the cars arriving before the morning peak.

In addition, it is also useful to know how much of the offered flexibility is utilized throughout the day. To quantize the degree of flexibility utilization, we use the Eflex and Tflex measures. Figure 6 shows, for a given time slot, the 
average Tflex and Eflex values for the sessions with arrivals in that slot (note that these sessions may extend until much later slots). The values are depicted for each behavioral cluster during weekdays vs. weekends. The empty sections in the plots indicate there were either no arrivals occurred, or the arrivals had zero idle times at these times of day. We list our observations for the Eflex and Tflex in the load flattening scenario, which essentially also apply qualitatively for the load balancing case.

For Tflex: In general, Tflex close to 1 means that charging lasts almost until the end of the sojourn. Yet, this does not mean that all charging is delayed (see the Eflex which is reasonably low, meaning that the SoC at $t_{B A U}$ is pretty high). We observe lower Tflex for arrivals at night and in the early morning (i.e., $0-6 \mathrm{am}$ ). The reason is that the sessions with arrival times in those slots 530 are responsible for the bulk of the load at those times, which is low compared to other slots, so there is a lower motivation to push their charging away and make use of flexibility. Any arrivals in the subsequent slots have their load shifted away from the peaks, hence, the Tflex value increases and approaches 1. Tflex starts to decrease again for the arrivals near midnight.

For Eflex: similar to Tflex, lower Eflex is observed for arrivals at night and early morning (i.e., $0-6 \mathrm{am}$ ) since the bulk of the load at those times is low and hence, there is little need for deferring the consumption. The Eflex in the late morning $(9-11 \mathrm{am})$ is lower than in the afternoon/evening. Note that the arrivals in the late morning are usually used to fill the afternoon valley, but the amount 540 of energy pushed into afternoon valley from morning peaks is lower compared to the amount of energy pushed into night valley (the night valley is deeper and requires more load to be filled). Additionally, the arrivals in the late morning are typically from the park to charge or the charge near work clusters: since their sojourn does not overlap with the night valley, their load cannot be used 545 to fill the night valley. Another interesting observation is the bell shape of Eflex after $12 \mathrm{pm}$ in the park to charge cluster for both weekends and weekdays, which peaks around $4 \mathrm{pm}$ and $6 \mathrm{pm}$ respectively. Note that since the sessions in this cluster have very small idle times, a larger portion of their energy consumption 
is deferred, but for shorter duration, to flatten the load. In the charge near home

cluster, we see a rather linear increase in Eflex. The sessions in this cluster offer much longer idle times compared to the park to charge cluster. By observing the $\mathrm{SoC}$ status of the sessions in this cluster, we find that for the sessions whose sojourns overlap with the evening peak, their charging usually stops during the peak hours and resumes in the night valley. That is the main reason for Tflex close to one but rather small Eflex for sessions with arrivals in the afternoon and evening.

\section{Summary and Conclusion}

Motivated by the lack of research in characterizing the flexibility stemming from EV charging sessions, in this paper we took the first step to (1) offer an in-depth analysis of the flexibility characteristics of a nearly 390k EV charging sessions and (2) propose flexibility measures to quantify its exploitation in two scenarios, load flattening and load balancing. Our contributions in this paper pave the way to more realistic evaluation and development of DR algorithms, which aim to not only exploit the flexibility but also to influence it more efficiently (through price-based or incentive-based schemes).

To fulfill our first objective (i.e., analysis of flexibility characteristics), we clustered the EV data in 2D space in terms of arrival and departure times using the DBSCAN algorithm. As such, we identified three behavioral clusters: charge near home, charge near work, and park to charge clusters. We then used box and violin plots to further analyze the characteristics of the charging sessions within each cluster and highlighted the differences among the clusters over weekends and weekdays in each season. A summery of our observations is listed here:

1. The three behavioral clusters differ substantially in their arrival times, sojourn times and the idle times. The park to charge cluster (which is the largest in terms of number of sessions, $62.86 \%$ of all sessions) has arrivals scattered throughout the day and the sessions in this cluster are 
characterized by very short idle times (averaging $48 \mathrm{~min}$ ). The charge near work cluster (27.84\% of all sessions) has predictable arrival times (around 6-9 am) and their sojourn times are typically less than 9 hours (with average idle time of $5 \mathrm{~h} 30 \mathrm{~min}$ ), hence, their charging usually takes place throughout the day. Finally, the sessions in charge near home cluster (9.3\% of all sessions), with arrivals typically in the evening until midnight, offer the longest idle times among the clusters (10 h on average). The charging for these sessions usually occurs at night.

2. Weekends and weekdays as well as seasonal changes impact the arrival times in all three clusters. In general, the arrival times are earlier in summer and spring in all the clusters. The arrivals are also earlier on weekdays compared to weekends. However, seasons have no substantial impact on the sojourn and idle times. Sessions in park to charge and charge near work clusters have shorter sojourn and idle times in the weekends whereas the sessions in the charge near home clusters have longer sojourn and idle times in the weekends compared to weekdays.

To fulfill our second objective (i.e., quantification of flexibility exploitation), we proposed two flexibility measures to quantify the percentage of the flexibility utilization and an algorithm to determine the amount and duration of the shifted energy. A summary of our analysis using the algorithm and the measures is as follows.

1. The flexibility exploitation is greatly influenced by the uncontrolled business as usual (BAU) load patterns, the distribution of arrival times, and the renewable energy generation patterns. The main motivation for exploitation of the flexibility in both load flattening and load balancing is to fill the valleys of the BAU load pattern. Hence, longer shifts are observed from the evening peaks compared to the morning peaks in the weekdays (since the nighttime valley is larger and deeper). Similarly, longer shifts are seen from Saturday peaks compared to Sunday peaks because the night valley between Saturdays and Sundays is bigger.

2. For arrivals in the afternoon until midnight, flexibility in terms of de- 
ferrable time is almost fully exploited to ensure the charging takes place in the nighttime (which corresponds to the lower demand). Yet, this does not imply that all the charging is delayed since the Eflex values are reasonably low, meaning that the $\mathrm{SoC}$ at the $\mathrm{BAU}$ charging completion time (i.e., $\left.t_{B A U}\right)$ is pretty high. Across the behavioral clusters, the offered flexibility in charge near work cluster is often used to fill the afternoon valley since these sessions are characterized by morning arrivals and their sojourn typically does not cover the night valley. Hence, their exploitation in terms of deferrable time and energy is typically lower compared to the arrivals in the other clusters which are usually in the afternoon. The sessions in the charge near home cluster are the better candidate to fill the night valley.

We conclude that the sessions in the charge near work cluster should be targeted to provide long enough flexibility to fill the afternoon valley. Any longer idle time would not be exploited (unless it is long enough to cover the night valley). The sessions in the charge near home cluster should be targeted to fill the night valley and for arrivals after midnight in this cluster, there is less need for longer idle times. Finally, in the park to charge cluster, it is recommended to target the arrivals in the afternoon to stimulate longer flexibility durations to fill the afternoon valley.

\section{References}

[1] F. Teng, M. Aunedi, G. Strbac, Benefits of flexibility from smart electrified transportation and heating in the future uk electricity system, Applied Energy 167 (2016) 420 - 431. doi:10.1016/j.apenergy .2015.10.028.

[2] E. S. Rigas, S. D. Ramchurn, N. Bassiliades, Managing electric vehicles in the smart grid using artificial intelligence: A survey, IEEE Transactions on Intelligent Transportation Systems 16 (4) (2015) 1619-1635. doi:10. 1109/TITS.2014.2376873. 
[3] Z. Yang, K. Li, A. Foley, Computational scheduling methods for integrating plug-in electric vehicles with power systems: A review, Renewable and Sustainable Energy Reviews 51 (2015) 396 - 416. doi:10.1016/j.rser. 2015.06 .007

[4] K. M. Tan, V. K. Ramachandaramurthy, J. Y. Yong, Integration of electric vehicles in smart grid: A review on vehicle to grid technologies and optimization techniques, Renewable and Sustainable Energy Reviews 53 (C) (2016) 720-732.

${ }_{645}^{6}$ [5] J. Hu, H. Morais, T. Sousa, M. Lind, Electric vehicle fleet management in smart grids: A review of services, optimization and control aspects, Renewable and Sustainable Energy Reviews 56 (2016) 1207 - 1226. doi: $10.1016 /$ j.rser.2015.12.014.

[6] I. Lampropoulos, G. M. A. Vanalme, W. L. Kling, A methodology for modeling the behavior of electricity prosumers within the smart grid, in: Proc. IEEE PES Innovative Smart Grid Technologies Conference Europe (ISGT Europe), 2010, pp. 1-8. doi:10.1109/ISGTEUROPE.2010.5638967

[7] K. Clement-Nyns, E. Haesen, J. Driesen, The impact of charging plug-in hybrid electric vehicles on a residential distribution grid, IEEE Transactions on Power Systems 25 (1) (2010) 371-380. doi:10.1109/TPWRS. 2009. 2036481

[8] P. Paevere, A. Higgins, Z. Ren, M. Horn, G. Grozev, C. McNamara, Spatiotemporal modelling of electric vehicle charging demand and impacts on peak household electrical load, Sustainability Science 9 (1) (2014) 61-76. doi:10.1007/s11625-013-0235-3.

[9] P. Grahn, K. Alvehag, L. Sder, PHEV utilization model considering typeof-trip and recharging flexibility, IEEE Transactions on Smart Grid 5 (1) (2014) 139-148. doi:10.1109/TSG.2013.2279022. 
[10] E. Pashajavid, M. Golkar, Non-gaussian multivariate modeling of plug-in electric vehicles load demand, International Journal of Electrical Power and

n Energy Systems 61 (2014) 197 - 207. doi:10.1016/j.ijepes.2014.03. 021.

[11] J. Soares, N. Borges, M. A. F. Ghazvini, Z. Vale, P. de Moura Oliveira, Scenario generation for electric vehicles' uncertain behavior in a smart city environment, Energy 111 (2016) 664-675. doi:10.1016/j.energy . 2016. 06.011

[12] E. Xydas, C. Marmaras, L. M. Cipcigan, N. Jenkins, S. Carroll, M. Barker, A data-driven approach for characterising the charging demand of electric vehicles: A UK case study, Applied Energy 162 (2016) 763 - 771. doi: $10.1016 / \mathrm{j}$. apenergy.2015.10.151.

[13] Y. B. Khoo, C.-H. Wang, P. Paevere, A. Higgins, Statistical modeling of electric vehicle electricity consumption in the victorian EV trial, australia, Transportation Research Part D: Transport and Environment 32 (2014) 263 - 277. doi:10.1016/j.trd.2014.08.017

[14] J. Brady, M. O’Mahony, Modelling charging profiles of electric vehicles based on real-world electric vehicle charging data, Sustainable Cities and Society 26 (2016) 203 - 216. doi:10.1016/j.scs.2016.06.014.

[15] J. Quiròs-Tortòs, L. F. Ochoa, S. W. Alnaser, T. Butler, Control of ev charging points for thermal and voltage management of lv networks, IEEE Transactions on Power Systems 31 (4) (2016) 3028-3039. doi: 10.1109/TPWRS.2015.2468062.

[16] A. Navarro-Espinosa, L. F. Ochoa, Probabilistic impact assessment of low carbon technologies in lv distribution systems, IEEE Transactions on Power Systems 31 (3) (2016) 2192-2203. doi:10.1109/TPWRS.2015.2448663.

[17] T. Franke, J. F. Krems, Understanding charging behaviour of electric ve- 
hicle users, Transportation Research Part F: Traffic Psychology and Behaviour 21 (2013) 75 - 89. doi:10.1016/j.trf.2013.09.002

[18] J. Spoelstra, Charging behaviour of dutch ev drivers, Master's thesis (2014).

[19] J. Quiròs-Tortòs, L. F. Ochoa, B. Lees, A statistical analysis of ev charging behavior in the uk, in: 2015 IEEE PES Innovative Smart Grid Technologies Latin America (ISGT LATAM), 2015, pp. 445-449. doi: 10.1109/ISGT-LA.2015.7381196.

[20] P. Richardson, M. Moran, J. Taylor, A. Maitra, A. Keane, Impact of electric vehicle charging on residential distribution networks: An irish demonstration initiative, in: 22nd International Conference and Exhibition on Electricity Distribution (CIRED 2013), 2013, pp. 1-4. doi: $10.1049 / \mathrm{cp} .2013 .0873$.

[21] J. Helmus, R. van den Hoed, Unraveling user type characteristics: Towards a taxonomy for charging infrastructure, in: Proc. 28th Int. Electric Vehicle Symp. and Exhibition (EVS 28), Goang, Korea, 2015, pp. 1211-1226.

[22] M. Aunedi, M. Woolf, G. Strbac, O. Babalola, M. Clark, Characteristic demand profiles of residential and commercial EV users and opportunities for smart charging, in: Proc. 23rd Int. Conf. Electricity Distribution (CIRED 2015), Lyon, France, 2015, pp. 1-5.

[23] E. C. Kara, J. S. Macdonald, D. Black, M. Brges, G. Hug, S. Kiliccote, Estimating the benefits of electric vehicle smart charging at non-residential locations: A data-driven approach, Applied Energy 155 (2015) 515 - 525. doi:10.1016/j.apenergy.2015.05.072.

[24] I. Pavi, T. Capuder, I. Kuzle, Value of flexible electric vehicles in providing 715 a spinning reserve services, Applied Energy 157 (2015) 60 - 74. doi:10. 1016/j.apenergy.2015.07.070.

[25] F. Salah, A. Schuller, M. Maurer, C. Weinhardt, Pricing of demand flexibility: Exploring the impact of electric vehicle customer diversity, in: 2016 
13th Int. Conf. on the European Energy Market (EEM), 2016, pp. 1-5. doi:10.1109/EEM.2016.7521202.

[26] M. Khederzadeh, Inherent potential of electrical vehicles to flatten the daily load curve in a microgrid, in: Proc. 23rd Int. Conf. Electricity Distribution (CIRED 2015), Lyon, France, 2015, pp. 1-5.

[27] A. Schuller, C. M. Flath, S. Gottwalt, Quantifying load flexibility of electric vehicles for renewable energy integration, Applied Energy 151 (2015) 335 - 344. doi:10.1016/j.apenergy.2015.04.004.

[28] C. Develder, N. Sadeghianpourhamami, M. Strobbe, N. Refa, Quantifying flexibility in EV charging as DR potential: Analysis of two real-world data sets, in: Proc. 7th IEEE Int. Conf. Smart Grid Communications (SmartGridComm 2016), Sydney, Australia, 2016, pp. 1-6.

[29] M. Ester, H.-P. Kriegel, J. Sander, X. Xu, A density-based algorithm for discovering clusters in large spatial databases with noise., in: Kdd, Vol. 96, 1996, pp. 226-231.

[30] G. Hamerly, C. Elkan, Learning the k in k-means, in: In Neural Information Processing Systems, MIT Press, 2003, p. 2003.

[31] K. Mets, F. De Turck, C. Develder, Distributed smart charging of electric vehicles for balancing wind energy, in: Proc. 3rd IEEE Int. Conf. Smart Grid Communications (SmartGridComm 2012), Tainan City, Taiwan, 2012, pp. 133-138. doi:10.1109/SmartGridComm.2012.6485972.

[32] N. Sadeghianpourhamami, T. Demeester, D. F. Benoit, M. Strobbe, C. Develder, Modeling and analysis of residential flexibility: Timing of (4) white good usage, Applied Energy 179 (2016) 790-805. doi:10.1016/j. apenergy.2016.07.012. 